Review

\title{
Lipid Transport and Metabolism in Healthy and Osteoarthritic Cartilage
}

\author{
Amanda Villalvilla ${ }^{1, *}$, Rodolfo Gómez ${ }^{2}$, Raquel Largo ${ }^{1}$ and Gabriel Herrero-Beaumont ${ }^{1}$ \\ 1 Osteoarticular Pathology Laboratory, IIS Fundación Jiménez Díaz, Madrid 28040, Spain; \\ E-Mails: rlargo@fjd.es (R.L.); gherrero@fjd.es (G.H.-B.) \\ 2 Musculoskeletal Research Group, Institute of Cellular Medicine, Newcastle University, \\ Newcastle upon Tyne NE2 4HH, UK; E-Mail: rodolfo.gomez@newcastle.ac.uk \\ * Author to whom correspondence should be addressed; E-Mail: amanda.villalvilla@fjd.es; \\ Tel.: +34-915-504-800; Fax: +34-915-442-636.
}

Received: 23 August 2013; in revised form: 8 October 2013 / Accepted: 8 October 2013 / Published: 16 October 2013

\begin{abstract}
Cartilage is an avascular tissue and cartilage metabolism depends on molecule diffusion from synovial fluid and subchondral bone. Thus, nutrient availability is limited by matrix permeability according to the size and charge of the molecules. Matrix composition limits the access of molecules to chondrocytes, determining cell metabolism and cartilage maintenance. Lipids are important nutrients in chondrocyte metabolism and are available for these cells through de novo synthesis but also through diffusion from surrounding tissues. Cartilage status and osteoarthritis development depend on lipid availability. This paper reviews lipid transport and metabolism in cartilage. We also analyze signalling pathways directly mediated by lipids and those that involve mTOR pathways, both in normal and osteoarthritic cartilage.
\end{abstract}

Keywords: chondrocyte; cartilage; osteoarthritis; lipid; cholesterol; nutrition

\section{Introduction}

Lipid content in cartilage has been studied for many years. In the 1960s, Stockwell found that lipids in articular cartilage account for $1 \%$, although this content was not modulated by age or sex [1]. While glucose is the main source of energy in chondrocytes [2], lipids in cartilage are necessary for cells as a source of energy but also to be incorporated as structural components and signalling molecules. 
Cholesterol and fatty acids are the lipids that have been most frequently linked to cartilage physiopathology [3]. However, less is known about how these molecules can reach the chondrocytes and, once there, how lipids affect chondrocyte metabolism.

Proteins necessary for fatty acid metabolism and cholesterol biosynthesis, such as acetyl-coenzyme A acetyltransferase 1 (ACAT1), cytochrome P450 oxidase, family 51, sub-family A, polypeptide 1 (CYP51A1), 3-hydroxy-3-methylglutaryl-coenzyme A synthase 1 (HMGCS) or low density lipoprotein receptor (LDLR), have been detected in human chondrocytes [4]. In addition, lipids in joint fluid can also penetrate into cartilage [5]. Therefore, lipids may be available for chondrocytes directly from synovial fluid or by de novo synthesis.

Nevertheless, cartilage is an avascular tissue, so lipids need to travel through its compact matrix reaching the cells, which represent only about $2 \%-5 \%$ of total tissue, for supplying the chondrocyte metabolism. Therefore to better understand the role of lipids in chondrocyte metabolism it is necessary to analyze how these molecules are supplied to chondrocytes and afterwards to study lipid effects on cell metabolism.

\section{Transporting Lipids through the Cartilage}

Articular cartilage matrix is composed of $10 \%-30 \%$ collagen and 3\%-10\% proteoglycans and other minor glycoproteins and lipids. Proteoglycans, with a high anionic charge, provide the matrix with osmotic properties that allow cartilage resistance to loading, while also interacting with collagen to establish a network [6,7]. Therefore, cartilage matrix is basically a high negative charged network with large swelling pressure and tensile stress, where water and dissolved electrolytes filling the pores account for between $60 \%$ and $85 \%$ of total weight [7]. This complex structure determines the ability of articular cartilage to resist compressive loads, but also determines how molecules penetrate this matrix.

Small neutral solutes can diffuse freely from joint fluid into cartilage, while ionic and larger molecules show altered movement through the matrix. As described above, cartilage matrix is negatively charged which means that small anionic molecules are partially excluded, and the opposite occurs with small cationic solutes. Moreover, the ability to penetrate the cartilage matrix depends on the valency, which determines molecule affinity for the matrix [8].

Proteoglycans are heterogeneously distributed throughout the matrix [9], and variations in tissue fixed charge density may influence solute diffusion, mostly for larger molecules [8]. The main role of proteoglycans in molecule diffusion was corroborated by digesting human articular cartilage with several proteases, although this process did not affect the circulation of small solutes. However, although collagen removal did not alter the diffusive properties of any molecule, cathepsin D and trypsin digestions increased the diffusion of large molecules [10].

Lipids are usually found in blood as lipoproteins or bound to carrier plasma proteins in order to increase their solubility. This way, lipids become part of a large complex which restricts their transport through cartilage since size is a determining factor for molecule diffusion [11]. However, as Arkill et al. [11] suggested, this is not always the case, since lauric acid dissociates from albumin in cartilage surface and independently diffuses into the cartilage. In fact, this fatty acid was detected in the entire cartilage, accumulating in the tidemark [11]. 
Despite a different matrix composition, mature and immature cartilage show similar permeability, at least for uncharged solutes [12]. However, mature joints present a calcified cartilage in the bone-cartilage interface that does not exist in immature joints. This natural barrier could strongly interfere with the diffusion of lipids and other nutrients from calcified to non-calcified cartilage. Taking into account that diffusion of large molecules through cartilage matrix may be restricted, it is very important to understand what the possible nutrient sources in the joint are. Several studies have been carried out considering two alternatives: diffusion from synovial fluid and interchange from subchondral bone (SB).

There are big controversies about the $\mathrm{SB}$ as a source providing nutrients such as lipids to the cartilage, with the calcified cartilage in the bone-cartilage interface of mature joints being an important point in this debate. No nutrient diffusion from SB has been found in mature rabbits [13-15]; however, transport of molecules from SB has been shown in horses and mice [16,17]. In fact, mice present non-mineralized regions in calcified cartilage, forming pores to allow solute transport between cartilage and SB in mature joints [16].

Regarding human cartilage, it was firstly suggested that vascular channels penetrating from SB allowed molecule diffusion through calcified cartilage in human mature joints [18]. More recently the chondro-osseous junction in mature human cartilage was described as a complex 3D structure which presents a clearly defined tidemark that follows uncalcified cartilage prolongations crossing calcified cartilage to contact with bone and bone marrow vessels, allowing molecular trafficking [19].

Unlike subchondral bone, synovial fluid is widely accepted as a main source of molecules for articular cartilage metabolism [20,21]. In fact, Wang et al. [20] found that synovial fluid, but not SB, provides enough nutrients to maintain cartilage structure and function in mature rabbits.

In this regard, it is noteworthy that joints are subjected to compression and cyclic loading in vivo, modifying molecule diffusion from synovial fluid. Although molecule diffusion occurs in the absence of mobilization, fluid stirring and convection and cyclic loading are important for the diffusion of small solutes but even more for large solutes [22-24].

\subsection{Osteoarthritis and Lipid Availability}

Osteoarthritis (OA) is characterized by high levels of proteases that degrade cartilage matrix [25]. As stated above, this process involves increased cartilage permeability, which in turn could contribute to OA evolution [10]. Matrix components synthesized by chondrocytes in an attempt to repair the damaged cartilage could be lost in the joint space, being an unproductive effort [10]. Moreover, deleterious agents such as toxins and immunoglobulins, usually excluded from the cartilage, could reach chondrocytes and induce metabolic changes [10]. In fact, OA synovial fluid presents high levels of plasma proteins, which could easily reach chondrocytes and induce proinflammatory responses [26] (Table 1).

Moreover, OA cartilage does not only receive high levels of molecules from synovial fluid due to an increase in an aberrant permeability. Pan et al. found that during OA the cross-talk between SB and articular cartilage is enhanced due to an increase in the number of vessels invading the calcified cartilage [27]. In turn, immobilization and disability due to pain in OA patients may also affect cartilage permeability and therefore nutrient availability for the chondrocytes [22] (Table 1). 
Table 1. Altered cartilage permeability during osteoarthritis (OA).

\begin{tabular}{llc}
\hline Altered cartilage permeability & Events related to OA & References \\
\hline \multirow{4}{*}{ Causes } & Increased protease activity & {$[25]$} \\
& Increased subchondral vessels invading calcified cartilage & {$[27]$} \\
& Joint immobilization & {$[22]$} \\
\hline \multirow{3}{*}{ Consequences } & Loss of matrix components in the joint space & {$[10]$} \\
& Access of deleterious agents (toxins, immunoglobulins) & {$[10]$} \\
& Access of proinflammatory plasma proteins & {$[26]$} \\
\hline
\end{tabular}

\section{Lipids in Normal Cartilage}

As stated above, human chondrocytes express several proteins for fatty acid metabolism and cholesterol biosynthesis. These molecules are up-regulated during chondrogenesis, indicating a high cholesterol biosynthesis in these cells [4]. In fact, cholesterol biosynthesis is required for expression of Ihh in rat chondrocytes and for normal growth plate chondrogenesis in rats [28,29].

On the other hand, leptin-like oxidized LDL (oxLDL) receptor 1 (LOX-1) [30] and Liver X receptor (LXR) [31] have been detected in human cartilage. These data indicate that chondrocytes are indeed able to modulate lipid homeostasis in the cartilage by sensing and transporting lipoproteins from the extracellular matrix.

Synovial fluid is an ultrafiltrate of plasma. However, plasma lipids are transported in large complexes so that human synovial fluid presents very low concentrations of lipoproteins in contrast to plasma [32,33]. It is important to note that the inflammation and vascular permeability of synovium determine the levels of lipoproteins in synovial fluid. Thereby, the ratio of synovial fluid to plasma of ApoA1, ApoB and Lp(a) is about 1.5 times higher in inflammatory arthritides like rheumatoid and psoriatic arthritis than in OA [33].

Despite being able to penetrate into cartilage, lipid content constitutes less than $1 \%$ of the total tissue weight [1]. High cholesterol content is found in chondrocyte cell membrane [34], indicating the importance of this lipid for structural maintenance in chondrocytes. In addition, the phytosterol stigmasterol is able to integrate in the chondrocyte membrane during culture [35]. On the other hand, palmitic, oleic and linoleic acids (16:0, 18:1n-9 and 18:2n-6) account for almost $85 \%$ of total fatty acid content in cartilage [36]. Moreover, their presence in cartilage is subjected to age-related changes. Studies both in humans and sheep demonstrated that fetal cartilage differs in lipid composition from mature cartilage. Indeed, human mature cartilage showed decreased monounsaturated and $\omega-3$ fatty acids, while saturated fatty acids were increased [37]. In addition, arachidonic acid (20:4n-6) is higher in fetal cartilage meanwhile linoleic acid (18:2n-6) increases in mature cartilage (Table 2) [37]. Moreover, cultured growth plate chondrocytes present higher content in lipids than cultured articular chondrocytes [38].

It is noteworthy that, besides physiological modification of lipid content, the fatty acid composition in cartilage may be modulated by dietary lipids intake $[39,40]$. Diets containing high levels of $\omega-3$ fatty acids lead to a decrease in arachidonic (20:4n-6) and linoleic acids (18:2n-6) and an increase in eicosapentaenoic acid (20:5n-3) in cartilage [39,40]. In addition, Nagao et al. demonstrated that extracellular fatty acids modulate intracellular lipid composition in cultured chondrocytes [41]. 
Table 2. Fatty acid composition in normal cartilage.

\begin{tabular}{llc}
\hline Cartilage maturation stage & Cartilage composition & References \\
\hline \multirow{2}{*}{ Mature cartilage } & $\uparrow$ saturated fatty acids & {$[37]$} \\
& $\uparrow$ linoleic acid & {$[37]$} \\
\hline \multirow{3}{*}{ Fetal cartilage } & $\uparrow \omega-3$ fatty acids & {$[37]$} \\
& $\uparrow$ mono-unsaturated fatty acids & {$[37]$} \\
& $\uparrow$ arachidonic acid & {$[37]$} \\
\hline
\end{tabular}

As stated above, cholesterol plays an important role as a structural molecule. However, the main effect of fatty acids in cartilage is through its conversion to eicosanoids. Both $\omega-3$ and $\omega-6$ fatty acids are substrates for cyclooxygenase and lipoxygenase enzymes, which synthesize prostaglandins and leukotrienes. These products derived from $\omega$-3 fatty acids show anti-inflammatory properties, while those derived from $\omega-6$ are pro-inflammatory and pro-thrombotic [42]; in fact, inhibition of cyclooxygenase results in less inflammatory mediators [43,44]. However, $\omega-6$ fatty acids are also important for membrane structure and function, so it is necessary to maintain an appropriate ratio of $\omega-6$ to $\omega-3$, with $4: 1$ to $1: 1$ recommended [45].

Phospholipids are also important for normal cartilage maintenance. Besides being cell membrane constituents, phospholipids are key molecules in synovial fluid, playing a main role in joint lubrication to protect cartilage surfaces. In fact, phosphatidylethanolamine, phosphatidylcholine and sphingomyelin are major components of the articular cartilage boundary lubricant [46]. In addition to the presence of these phospholipids in the joint fluid, free fatty acids may be incorporated by chondrocytes into phosphatidylcholine (PC), phosphatidylethanolamine (PE), phosphatidylinositol (PI) and triacylglycerol (TG) $[41,47]$.

Therefore, lipids are essential for cartilage physiology, and modifications in their availability and metabolism may have pathological consequences.

\section{Lipids in Osteoathritic Cartilage}

In recent years, OA has been linked to metabolic syndrome, which is characterized by dyslipidemia [48]. In fact, several studies have found an association between hypercholesterolemia and OA [49-51]. Therefore, some epidemiological studies have tried to elucidate the possible relationship between statin treatment to reduce serum cholesterol levels and OA development. However, the interpretation of the results of these studies presents some limitations regarding the evaluation of OA progression [52], leading to different conclusions. Clockaerts et al. [53] and Kadam et al. [54] found a reduced progression of knee OA in statin users; on the contrary, Riddle et al. [55] did not find an improvement in knee pain, function or structural progression, while Beattie et al. [56] concluded that statins did not influence the progression of an established OA.

Despite these controversies, the potential of statins to reduce OA has been observed in vitro and in animal models. In fact, statin treatment reduced OA progression in rats [57] and mice [58], and decreased pro-inflammatory and catabolic mediators in cultured chondrocytes [59-61]. However, cholesterol biosynthesis plays an important role in chondrogenesis [28,29]. Accordingly, these beneficial effects of statins have been related to reduced protein geranylgeranylation rather than inhibition of cholesterol synthesis itself $[60,62]$. 
Several studies have shown the beneficial effects of $\omega$-3 fatty acids in inflammatory diseases such as rheumatoid arthritis (RA), recommending their use as part of the normal diet of patients [42]. However, only a few studies, without conclusive results, have been carried out in OA. Treating OA patients with cod liver oil, which contains high levels of eicosapentanoic acid (20:5n-3), in addition to non-steroidal anti-inflammatory drugs (NSAIDs) for 24 weeks showed no benefits versus olive oil [63]. However, the anti-inflammatory properties of several olive oil components could have attenuated the differences due to fatty acid composition [64]. In keeping with the lack of cod liver oil effect, Wang et al. found a correlation between $\omega-6$ fatty acids intake and development of bone marrow lesions, but not with cartilage volume or damage [65]. In contrast, a recent study showed that total $\omega-3$ fatty acids and the specific docosahexanoic acid (22:6n-3) were inversely correlated with patellofemoral cartilage loss, while $\omega-6$ fatty acids showed no association [66]. Moreover, an animal model of spontaneous OA fed with a $\omega-3$ fatty acids-enriched diet showed a lower pathology score, with increased glycosaminoglycan content, reduced denatured type II collagen, and reduced MMP-2 activity [67]. Very recently, Huang et al. found that decreasing $\omega-6$ to $\omega-3$ ratio by endogenous conversion of $\omega-6$ to $\omega$-3 fatty acids in mice delayed OA development [68]. Therefore, a beneficial effect of $\omega-3$ fatty acids for OA cartilage may exist, although further in vivo and in vitro studies are needed to demonstrate it.

As stated above, phospholipids are important in joint lubrication. However, most of the phospholipids present in synovial fluid appear increased in OA, which could assist OA pathogenesis in modulating inflammatory responses [69]. In addition, high levels of phospholipase A2 are present in OA synovial fluid [70], where the main sources of this enzyme are the chondrocytes [71]. In fact, cytokines present in OA joints such as IL-1 are able to activate phospholipase A2 in chondrocytes, suggesting that this enzyme may play a role in the OA development [72]. Moreover, the activity of phospholipase A2 in OA synovial fluid could contribute to a release of fatty acids, which penetrate the cartilage matrix more easily than large complexes [11].

As with synovial fluid, lipid accumulation has been described in articular cartilage during OA development, despite the fact that diet association in OA still needs clarification. In fact, using imaging mass spectrometry, Cillero-Pastor et al. [34] have recently shown that cholesterol and fatty acids specifically accumulate in the superficial area of OA cartilage. Moreover, reduced expression of LXR and ApoA1 has been described in human OA cartilage, leading to impaired cholesterol efflux and finally intracellular lipid deposits in OA chondrocytes [31]. In fact, ApoA $1^{-/}$mice spontaneously developed OA when fed a western-type diet due to alterations in HDL metabolism [73]. Changes in cartilage lipid composition are correlated with disease severity, with increases in total fatty acid of $440 \%$ in the advanced stages of OA [36]. Lippiello et al. suggested that high levels of arachidonic acid (20:4n-6) in OA could be related to the elevated eicosanoid synthesis usually found in this disease [36]. Therefore, altered lipid metabolism could be a risk factor and/or consequence of OA [34].

Lipid deposition in cartilage during OA has more consequences than merely accumulation itself. $\mathrm{OA}$ is characterized by an increase in reactive oxygen species, which are responsible for lipid peroxidation [74]; thus, lipid peroxidation products are usually found in joints from these patients $[75,76]$. Several studies have reported that these molecules contribute to cartilage degradation and OA pathogenesis, since they induce collagen oxidation and cleavage and MMP-13 
activity [74,76]. Thereby, antioxidant treatment may be useful for OA not only by reducing reactive oxygen species [77] but also lipid peroxidation.

\section{Lipids and Chondrocyte Metabolism}

Besides its structural role, cholesterol is also an important signal in chondrocyte biology. LXR is a sensor of oxygenated cholesterol derivatives, which activates transcription of important genes to protect cells from cholesterol overload. This receptor is modulated during chondrocyte differentiation and regulates cholesterol homeostasis during this process [78]. In fact, cholesterol signalling stimulates in vitro chondrocyte hypertrophy through nuclear receptor retinoid related orphan receptor- $\alpha$ (Ror- $\alpha$ ) expression [79], and it is also necessary for apoptosis protection and Ihh expression during chondrogenesis and growth plate development [28,29] (Figure 1).

Figure 1. Summary of lipid actions in cartilage metabolism and response to stress. Green arrows indicate activation and red arrows inhibition of the processes shown in the squares. References are indicated in brackets.

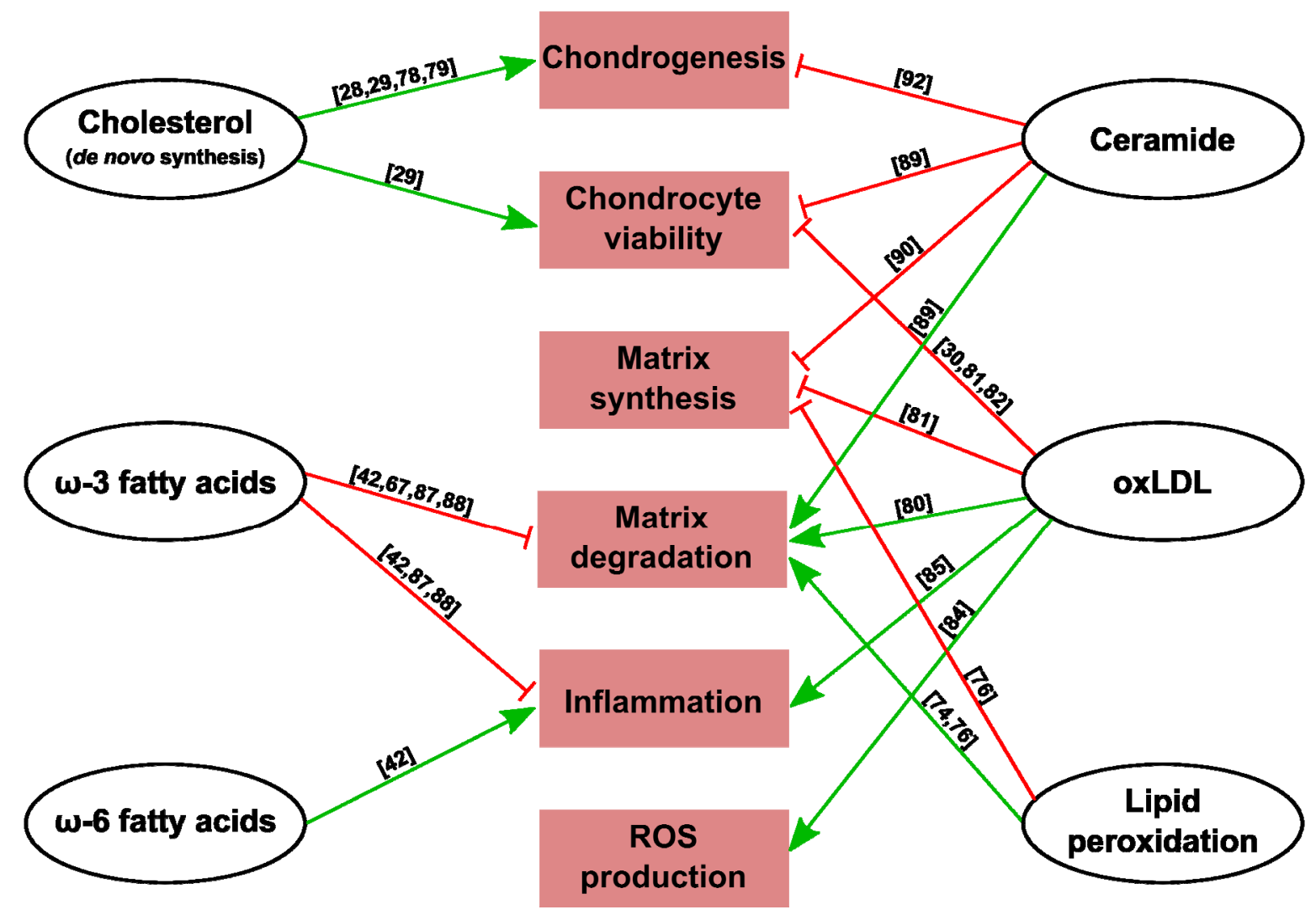

As stated above, OA chondrocytes express LOX-1, a scavenger receptor for oxLDL. oxLDL has been detected in human OA and RA cartilage [80,81], and stimulates senescence in human chondrocytes in vitro [82] by decreasing cell viability [30,81]. Moreover, this molecule induces reactive oxygen species production and hypertrophic-like changes in bovine chondrocytes [83,84], reduces proteoglycan synthesis in human chondrocytes [81] and increases MMP-3 production in human cartilage explants [80] and MCP-1 expression in human chondrocytes [85] (Figure 1). 
The effects of fatty acids on chondrocyte metabolism support the potential benefits of adequate lipid content in diet. During in vitro experiments, bovine chondrocytes rapidly incorporated both arachidonic acid (20:4n-6) and oleic acid (18:1n-9). However, only arachidonic acid elicited metabolic changes, with increased matrix and prostaglandin synthesis [86]. Moreover, unlike arachidonic (20:4n-6), linoleic (18:2n-6), oleic (18;1n-9) and palmitic (16:0) acids, $\omega-3$ fatty acids decrease proteinases involved in cartilage matrix degradation, COX-2, IL-1 $\alpha$, IL-1 $\beta$ and TNF $\alpha$ expression in chondrocytes [87,88] (Figure 1). However, rats fed 10\% menhaden oil, which contains high levels of $\omega$-3 fatty acids, showed a decrease in linoleic (18:2n-6) and arachidonic (20:4n-6) acids together with low proteoglycan synthesis and irregularities in cartilage [39]. This study points to the importance of an appropriate $\omega-6$ to $\omega-3$ ratio, where a high ratio may be as harmful as a very low one.

Ceramide is another lipid that is increased in the synovial fluid of OA patients [69]. Ceramide might play a mediatory role in cartilage loss during OA due to increased chondrocyte apoptosis and by inducing proteoglycan degradation [89]. High levels of endogenous ceramide have been demonstrated to disrupt cartilage matrix homeostasis, resulting in down-regulation of type II collagen in articular cartilage [90,91]. In fact, acid ceramidase, by degrading ceramide, alters sphingolipid metabolism and improves chondrogenesis [92] (Figure 1).

The mammalian target of rapamycin (mTOR) signalling pathway integrates both intracellular and extracellular signals and serves as a central regulator of cell metabolism, growth, proliferation and survival. Nutrients and energy status modulate mTOR signalling, leading to modifications in protein and lipid synthesis, lipid and glucose metabolism and autophagy [93]. Although mTOR is a critical regulator of lipid biosynthesis, its role as a lipid-sensing molecule has been studied to a lesser extent [94]. Palmitic acid (16:0) induces insulin resistance through activation of mTOR in skeletal muscle cells and hepatocytes [95,96], and a high-fat diet also induced insulin resistance in skeletal muscle in rats by mTOR activation [96]. In addition, arachidonic acid (20:4n-6) activates amino acid-independent mTOR signalling in breast cancer cells, inducing proliferation and angiogenesis [97]; however, ceramide induces autophagy by inhibiting mTOR signalling in several cell types [98]. mTOR has also been implied in macrophages' intracellular cholesterol homeostasis, regulating the expression of important genes for cholesterol metabolism such as ABCA1, LOX-1 and LXR [99]. In addition, cholesterol membrane content is a regulator of mTOR signalling in endothelial cells, since alteration in cholesterol distribution in plasma membrane blunts mTOR pathway [100].

Therefore, mTOR is related to lipid-induced responses in different cell types. This pathway can induce insulin resistance and modulate cell proliferation and angiogenesis in response to specific fatty acids, and membrane cholesterol also seems to be necessary for mTOR signalling. However, little is known about this signalling pathway in chondrocytes. It has been implicated in the process of chondrocyte differentiation, through modulation of autophagy and Ihh expression [101,102]. In fact, inhibition of mTOR by rapamycin prevents chondrocyte differentiation, showing an important role for mTOR in this process [79]. Autophagy has been proposed as a protective mechanism in normal cartilage; this way, aging and OA-related autophagy loss could be involved in cartilage damage during these situations [103]. Accordingly, autophagy induction by mTOR blockade using rapamycin in OA mice reduced disease severity, as shown by less cartilage degradation [104]. In contrast, Sasaki et al. found increased autophagy in OA chondrocytes, however, they also suggested that rapamycin-induced autophagy is a protective mechanism from stresses [105]. According to results in 
other cell types, arachidonic acid (20:4n-6) has been described as an activator of mTOR signalling in mice chondrocytes, while docosahexaenoic acid (22:6n-3) reverts this effect [68]. This recent study further confirms the beneficial role of $\omega-3$ fatty acids in chondrocytes, inhibiting mTOR and activating autophagy as a protective mechanism for cartilage maintenance [68].

\section{Conclusions}

Lipids such as phospholipids, cholesterol and fatty acids are present in articular cartilage and synovial fluid. Chondrocytes can synthesize these molecules, however, dietary lipids may also reach the cartilage, modify its composition and be incorporated in chondrocyte metabolism and structures. In fact, impaired cholesterol efflux and intracellular lipid deposits, as well as fatty acid accumulation in cartilage, have been related to OA development.

Several studies have suggested that anti-inflammatory and anti-catabolic properties of $\omega-3$ fatty acids, as well as their capacity to inhibit mTOR signalling and promote autophagy, may be used to treat OA symptoms and progression. Therefore, an appropriate lipid intake could contribute to improving lipid balance in cartilage, preventing chondrocyte metabolism alterations and cartilage damage.

\section{Acknowledgments}

This work was supported by research grants from Fondo de Investigación Sanitaria (FIS) (PS09/01625 and PI12/00144). Amanda Villalvilla is the recipient of a fellowship from the Fundación Conchita Rábago. Rodolfo Gómez is the recipient of a Sara Borrell contract from FIS. Raquel Largo's work was funded by the Instituto de Salud Carlos III through a research staff stabilization program.

\section{Conflicts of Interest}

The authors declare no conflict of interest.

\section{References}

1. Stockwell, R.A. Lipid content of human costal and articular cartilage. Ann. Rheum. Dis. 1967, 26, 481-486.

2. Otte, P. Basic cell metabolism of articular cartilage. Manometric studies. Z. Rheumatol. 1991, 50, 304-312.

3. Gkretsi, V.; Simopoulou, T.; Tsezou, A. Lipid metabolism and osteoarthritis: Lessons from atherosclerosis. Prog. Lipid Res. 2011, 50, 133-140.

4. Bernstein, P.; Sticht, C.; Jacobi, A.; Liebers, C.; Manthey, S.; Stiehler, M. Expression pattern differences between osteoarthritic chondrocytes and mesenchymal stem cells during chondrogenic differentiation. Osteoarthr. Cartil. 2010, 18, 1596-1607.

5. Ghadially, F.N.; Mehta, P.N.; Kirkaldy-Willis, W.H. Ultrastructure of articular cartilage in experimentally produced lipoarthrosis. J. Bone Jt. Surg. Am. 1970, 52, 1147-1158.

6. Roughley, P.J.; Lee, E.R. Cartilage proteoglycans: Structure and potential functions. Microsc. Res. Tech. 1994, 28, 385-397. 
7. Cohen, N.P.; Foster, R.J.; Mow, V.C. Composition and dynamics of articular cartilage: Structure, function, and maintaining healthy state. J. Orthop. Sports Phys. Ther. 1998, 28, 203-215.

8. Maroudas, A. Transport of solutes through cartilage: Permeability to large molecules. J. Anat. 1976, 122, 335-347.

9. Gonsalves, M.; Macpherson, J.V.; O’Hare, D.; Winlove, C.P.; Unwin, P.R. High resolution imaging of the distribution and permeability of methyl viologen dication in bovine articular cartilage using scanning electrochemical microscopy. Biochim. Biophys. Acta 2001, 1524, 66-74.

10. Lotke, P.A.; Granda, J.L. Alterations in the permeability of articular cartilage by proteolytic enzymes. Arthritis Rheum. 1972, 15, 302-308.

11. Arkill, K.P.; Winlove, C.P. Fatty acid transport in articular cartilage. Arch. Biochem. Biophys. 2006, 456, 71-78.

12. Torzilli, P.A.; Grande, D.A.; Arduino, J.M. Diffusive properties of immature articular cartilage. J. Biomed. Mater. Res. 1998, 40, 132-138.

13. Honner, R.; Thompson, R.C. The nutritional pathways of articular cartilage. An autoradiographic study in rabbits using 35S injected intravenously. J. Bone Jt. Surg. Am. 1971, 53, 742-748.

14. Hodge, J.A.; McKibbin, B. The nutrition of mature and immature cartilage in rabbits. An autoradiographic study. J. Bone Jt. Surg. Br. 1969, 51, 140-147.

15. Ogata, K.; Whiteside, L.A.; Lesker, P.A. Subchondral route for nutrition to articular cartilage in the rabbit. Measurement of diffusion with hydrogen gas in vivo. J. Bone Jt. Surg. Am. 1978, 60, 905-910.

16. Pan, J.; Zhou, X.; Li, W.; Novotny, J.E.; Doty, S.B.; Wang, L. In situ measurement of transport between subchondral bone and articular cartilage. J. Orthop. Res. 2009, 27, 1347-1352.

17. Arkill, K.P.; Winlove, C.P. Solute transport in the deep and calcified zones of articular cartilage. Osteoarthr. Cartil. 2008, 16, 708-714.

18. Greenwald, A.S.; Haynes, D.W. A pathway for nutrients from the medullary cavity to the articular cartilage of the human femoral head. J. Bone Jt. Surg. Br. 1969, 51, 747-753.

19. Lyons, T.J.; McClure, S.F.; Stoddart, R.W.; McClure, J. The normal human chondro-osseous junctional region: Evidence for contact of uncalcified cartilage with subchondral bone and marrow spaces. BMC Musculoskelet. Disord. 2006, doi:10.1186/1471-2474-7-52.

20. Wang, Y.; Wei, L.; Zeng, L.; He, D.; Wei, X. Nutrition and degeneration of articular cartilage. Knee Surg. Sports Traumatol. Arthrosc. 2012, 21, 1751-1762.

21. Levick, J.R. Microvascular architecture and exchange in synovial joints. Microcirculation 1995, 2, 217-233.

22. Maroudas, A.; Bullough, P.; Swanson, S.A.; Freeman, M.A. The permeability of articular cartilage. J. Bone Jt. Surg. Br. 1968, 50, 166-177.

23. Garcia, A.M.; Frank, E.H.; Grimshaw, P.E.; Grodzinsky, A.J. Contributions of fluid convection and electrical migration to transport in cartilage: Relevance to loading. Arch. Biochem. Biophys. 1996, 333, 317-325.

24. O'Hara, B.P.; Urban, J.P.; Maroudas, A. Influence of cyclic loading on the nutrition of articular cartilage. Ann. Rheum. Dis. 1990, 49, 536-539.

25. Appleton, C.T.G.; Pitelka, V.; Henry, J.; Beier, F. Global analyses of gene expression in early experimental osteoarthritis. Arthritis Rheum. 2007, 56, 1854-1868. 
26. Sohn, D.H.; Sokolove, J.; Sharpe, O.; Erhart, J.C.; Chandra, P.E.; Lahey, L.J.; Lindstrom, T.M.; Hwang, I.; Boyer, K.A.; Andriacchi, T.P.; et al. Plasma proteins present in osteoarthritic synovial fluid can stimulate cytokine production via Toll-like receptor 4. Arthritis Res. Ther. 2012, doi:10.1186/ar3555.

27. Pan, J.; Wang, B.; Li, W.; Zhou, X.; Scherr, T.; Yang, Y.; Price, C.; Wang, L. Elevated cross-talk between subchondral bone and cartilage in osteoarthritic joints. Bone 2012, 51, 212-217.

28. Wu, S.; de Luca, F. Role of cholesterol in the regulation of growth plate chondrogenesis and longitudinal bone growth. J. Biol. Chem. 2004, 279, 4642-4647.

29. Aguilar, A.; Wu, S.; de Luca, F. P450 oxidoreductase expressed in rat chondrocytes modulates chondrogenesis via cholesterol- and Indian hedgehog-dependent mechanisms. Endocrinology 2009, 150, 2732-2739.

30. Simopoulou, T.; Malizos, K.N.; Tsezou, A. Lectin-like oxidized low density lipoprotein receptor 1 (LOX-1) expression in human articular chondrocytes. Clin. Exp. Rheumatol. 2007, 25, 605-612.

31. Tsezou, A.; Iliopoulos, D.; Malizos, K.N.; Simopoulou, T. Impaired expression of genes regulating cholesterol efflux in human osteoarthritic chondrocytes. J. Orthop. Res. 2010, 28, 1033-1039.

32. Prete, P.E.; Gurakar-Osborne, A.; Kashyap, M.L. Synovial fluid lipids and apolipoproteins: A contemporary perspective. Biorheology 1995, 32, 1-16.

33. Oliviero, F.; Lo Nigro, A.; Bernardi, D.; Giunco, S.; Baldo, G.; Scanu, A.; Sfriso, P.; Ramonda, R.; Plebani, M.; Punzi, L. A comparative study of serum and synovial fluid lipoprotein levels in patients with various arthritides. Clin. Chim. Acta 2012, 413, 303-307.

34. Cillero-Pastor, B.; Eijkel, G.; Kiss, A.; Blanco, F.J.; Heeren, R.M.A. Time-of-flight secondary ion mass spectrometry-based molecular distribution distinguishing healthy and osteoarthritic human cartilage. Anal. Chem. 2012, 84, 8909-8916.

35. Gabay, O.; Sanchez, C.; Salvat, C.; Chevy, F.; Breton, M.; Nourissat, G.; Wolf, C.; Jacques, C.; Berenbaum, F. Stigmasterol: A phytosterol with potential anti-osteoarthritic properties. Osteoarthr. Cartil. 2010, 18, 106-116.

36. Lippiello, L.; Walsh, T.; Fienhold, M. The association of lipid abnormalities with tissue pathology in human osteoarthritic articular cartilage. Metabolism 1991, 40, 571-576.

37. Cleland, K.A.; James, M.J.; Neumann, M.A.; Gibson, R.A.; Cleland, L.G. Differences in fatty acid composition of immature and mature articular cartilage in humans and sheep. Lipids 1995, 30, 949-953.

38. Le Lous, M.; Corvol, M.T.; Maroteaux, P. Lipid composition of two types of chondrocytes in primary culture. Calcif. Tissue Int. 1981, 33, 403-407.

39. Lippiello, L.; Fienhold, M.; Grandjean, C. Metabolic and ultrastructural changes in articular cartilage of rats fed dietary supplements of omega-3 fatty acids. Arthritis Rheum. 1990, 33, 1029-1036.

40. Xu, H.; Watkins, B.A.; Adkisson, H.D. Dietary lipids modify the fatty acid composition of cartilage, isolated chondrocytes and matrix vesicles. Lipids 1994, 29, 619-625.

41. Nagao, M.; Ishii, S.; Murata, Y.; Akino, T. Effect of extracellular fatty acids on lipid metabolism in cultured rabbit articular chondrocytes. J. Orthop. Res. 1991, 9, 341-347. 
42. Lopez, H.L. Nutritional interventions to prevent and treat osteoarthritis. Part I: Focus on fatty acids and macronutrients. PMR 2012, 4, S145-S154.

43. Alvarez-Soria, M.A.; Largo, R.; Santillana, J.; Sánchez-Pernaute, O.; Calvo, E.; Hernández, M.; Egido, J.; Herrero-Beaumont, G. Long term NSAID treatment inhibits COX-2 synthesis in the knee synovial membrane of patients with osteoarthritis: Differential proinflammatory cytokine profile between celecoxib and aceclofenac. Ann. Rheum. Dis. 2006, 65, 998-1005.

44. Alvarez-Soria, M.A.; Herrero-Beaumont, G.; Moreno-Rubio, J.; Calvo, E.; Santillana, J.; Egido, J.; Largo, R. Long-term NSAID treatment directly decreases COX-2 and mPGES-1 production in the articular cartilage of patients with osteoarthritis. Osteoarthr. Cartil. 2008, 16, 1484-1493.

45. Simopoulos, A.P. The importance of the $\omega-6 / \omega-3$ fatty acid ratio in cardiovascular disease and other chronic diseases. Exp. Biol. Med. 2008, 233, 674-688.

46. Sarma, A.V.; Powell, G.L.; LaBerge, M. Phospholipid composition of articular cartilage boundary lubricant. J. Orthop. Res. 2001, 19, 671-676.

47. Nagao, M.; Ishii, S.; Kitamura, K.; Akino, T. Arachidonic acid metabolism in articular chondrocytes. Clin. Orthop. Relat. Res. 1991, 271, 288-295.

48. Zhuo, Q.; Yang, W.; Chen, J.; Wang, Y. Metabolic syndrome meets osteoarthritis. Nat. Rev. Rheumatol. 2012, 8, 729-737.

49. Al-Arfaj, A.S. Radiographic osteoarthritis and serum cholesterol. Saudi Med. J. 2003, 24, 745-747.

50. Stürmer, T.; Sun, Y.; Sauerland, S.; Zeissig, I.; Günther, K.P.; Puhl, W.; Brenner, H. Serum cholesterol and osteoarthritis. The baseline examination of the Ulm Osteoarthritis Study. J. Rheumatol. 1998, 25, 1827-1832.

51. Hart, D.J.; Doyle, D.V.; Spector, T.D. Association between metabolic factors and knee osteoarthritis in women: The Chingford Study. J. Rheumatol. 1995, 22, 1118-1123.

52. Conaghan, P.G. The effects of statins on osteoarthritis structural progression: Another glimpse of the Holy Grail? Ann. Rheum. Dis. 2012, 71, 633-634.

53. Clockaerts, S.; van Osch, G.J.V.M.; Bastiaansen-Jenniskens, Y.M.; Verhaar, J.A.N.; van Glabbeek, F.; van Meurs, J.B.; Kerkhof, H.J.M.; Hofman, A.; Stricker, B.H.C.; Bierma-Zeinstra, S.M. Statin use is associated with reduced incidence and progression of knee osteoarthritis in the Rotterdam study. Ann. Rheum. Dis. 2012, 71, 642-647.

54. Kadam, U.T.; Blagojevic, M.; Belcher, J. Statin use and clinical osteoarthritis in the general population: A longitudinal study. J. Gen. Intern. Med. 2013, 28, 943-949.

55. Riddle, D.L.; Moxley, G.; Dumenci, L. Associations between statin use and changes in pain, function and structural progression: A longitudinal study of persons with knee osteoarthritis. Ann. Rheum. Dis. 2013, 72, 196-203.

56. Beattie, M.S.; Lane, N.E.; Hung, Y.-Y.; Nevitt, M.C. Association of statin use and development and progression of hip osteoarthritis in elderly women. J. Rheumatol. 2005, 32, 106-110.

57. Aktas, E.; Sener, E.; Gocun, P.U. Mechanically induced experimental knee osteoarthritis benefits from anti-inflammatory and immunomodulatory properties of simvastatin via inhibition of matrix metalloproteinase-3. J. Orthop. Traumatol. 2011, 12, 145-151.

58. Yudoh, K.; Karasawa, R. Statin prevents chondrocyte aging and degeneration of articular cartilage in osteoarthritis (OA). Aging 2010, 2, 990-998. 
59. Dombrecht, E.J.; van Offel, J.F.; Bridts, C.H.; Ebo, D.G.; Seynhaeve, V.; Schuerwegh, A.J.; Stevens, W.J.; de Clerck, L.S. Influence of simvastatin on the production of pro-inflammatory cytokines and nitric oxide by activated human chondrocytes. Clin. Exp. Rheumatol. 2007, 25, 534-539.

60. Barter, M.J.; Hui, W.; Lakey, R.L.; Catterall, J.B.; Cawston, T.E.; Young, D.A. Lipophilic statins prevent matrix metalloproteinase-mediated cartilage collagen breakdown by inhibiting protein geranylgeranylation. Ann. Rheum. Dis. 2010, 69, 2189-2198.

61. Lazzerini, P.E.; Capecchi, P.L.; Nerucci, F.; Fioravanti, A.; Chellini, F.; Piccini, M.; Bisogno, S.; Marcolongo, R.; Laghi Pasini, F. Simvastatin reduces MMP-3 level in interleukin $1 \beta$ stimulated human chondrocyte culture. Ann. Rheum. Dis. 2004, 63, 867-869.

62. Sverdrup, F.M.; Yates, M.P.; Vickery, L.E.; Klover, J.A.; Song, L.R.-H.; Anglin, C.P.; Misko, T.P. Protein geranylgeranylation controls collagenase expression in osteoarthritic cartilage. Osteoarthr. Cartil. 2010, 18, 948-955.

63. Stammers, T.; Sibbald, B.; Freeling, P. Efficacy of cod liver oil as an adjunct to non-steroidal anti-inflammatory drug treatment in the management of osteoarthritis in general practice. Ann. Rheum. Dis. 1992, 51, 128-129.

64. Iacono, A.; Gómez, R.; Sperry, J.; Conde, J.; Bianco, G.; Meli, R.; Gómez-Reino, J.J.; Smith, A.B.; Gualillo, O. Effect of oleocanthal and its derivatives on inflammatory response induced by lipopolysaccharide in a murine chondrocyte cell line. Arthritis Rheum. 2010, 62, 1675-1682.

65. Wang, Y.; Wluka, A.E.; Hodge, A.M.; English, D.R.; Giles, G.G.; O’Sullivan, R.; Cicuttini, F.M. Effect of fatty acids on bone marrow lesions and knee cartilage in healthy, middle-aged subjects without clinical knee osteoarthritis. Osteoarthr. Cartil. 2008, 16, 579-583.

66. Baker, K.R.; Matthan, N.R.; Lichtenstein, A.H.; Niu, J.; Guermazi, A.; Roemer, F.; Grainger, A.; Nevitt, M.C.; Clancy, M.; Lewis, C.E.; et al. Association of plasma n-6 and n-3 polyunsaturated fatty acids with synovitis in the knee: The MOST study. Osteoarthr. Cartil. 2012, 20, 382-387.

67. Knott, L.; Avery, N.C.; Hollander, A.P.; Tarlton, J.F. Regulation of osteoarthritis by $\omega-3$ (n-3) polyunsaturated fatty acids in a naturally occurring model of disease. Osteoarthr. Cartil. 2011, 19, 1150-1157.

68. Huang, M.-J.; Wang, L.; Jin, D.; Zhang, Z.-M.; Chen, T.-Y.; Jia, C.-H.; Wang, Y.; Zhen, X.-C.; Huang, B.; Yan, B.; et al. Enhancement of the synthesis of n-3 PUFAs in fat-1 transgenic mice inhibits mTORC1 signalling and delays surgically induced osteoarthritis in comparison with wild-type mice. Ann. Rheum. Dis. 2013, doi:10.1136/annrheumdis-2013-203231.

69. Kosinska, M.K.; Liebisch, G.; Lochnit, G.; Wilhelm, J.; Klein, H.; Kaesser, U.; Lasczkowski, G.; Rickert, M.; Schmitz, G.; Steinmeyer, J. A lipidomic study of phospholipid classes and species in human synovial fluid. Arthritis Rheum. 2013, 65, 2323-2333.

70. Parks, T.P.; Lukas, S.; Hoffman, A.F. Purification and characterization of a phospholipase A2 from human osteoarthritic synovial fluid. Adv. Exp. Med. Biol. 1990, 275, 55-81.

71. Pruzanski, W.; Bogoch, E.; Stefanski, E.; Wloch, M.; Vadas, P. Enzymatic activity and distribution of phospholipase A2 in human cartilage. Life Sci. 1991, 48, 2457-2462.

72. Chang, J.; Gilman, S.C.; Lewis, A.J. Interleukin 1 activates phospholipase A2 in rabbit chondrocytes: A possible signal for IL 1 action. J. Immunol. 1986, 136, 1283-1287. 
73. Triantaphyllidou, I.-E.; Kalyvioti, E.; Karavia, E.; Lilis, I.; Kypreos, K.E.; Papachristou, D.J. Perturbations in the HDL metabolic pathway predispose to the development of osteoarthritis in mice following long-term exposure to western-type diet. Osteoarthr. Cartil. 2013, 21, 322-330.

74. Tiku, M.L.; Shah, R.; Allison, G.T. Evidence linking chondrocyte lipid peroxidation to cartilage matrix protein degradation. Possible role in cartilage aging and the pathogenesis of osteoarthritis. J. Biol. Chem. 2000, 275, 20069-20076.

75. Shah, R.; Raska, K.; Tiku, M.L. The presence of molecular markers of in vivo lipid peroxidation in osteoarthritic cartilage: A pathogenic role in osteoarthritis. Arthritis Rheum. 2005, 52, 2799-2807.

76. Morquette, B.; Shi, Q.; Lavigne, P.; Ranger, P.; Fernandes, J.C.; Benderdour, M. Production of lipid peroxidation products in osteoarthritic tissues: New evidence linking 4-hydroxynonenal to cartilage degradation. Arthritis Rheum. 2006, 54, 271-281.

77. Roman-Blas, J.A.; Contreras-Blasco, M.A.; Largo, R.; Alvarez-Soria, M.A.; Castañeda, S.; Herrero-Beaumont, G. Differential effects of the antioxidant $N$-acetylcysteine on the production of catabolic mediators in IL-1 $\beta$-stimulated human osteoarthritic synoviocytes and chondrocytes. Eur. J. Pharmacol. 2009, 623, 125-131.

78. Gentili, C.; Tutolo, G.; Pianezzi, A.; Cancedda, R.; Descalzi Cancedda, F. Cholesterol secretion and homeostasis in chondrocytes: $\mathrm{A}$ liver $\mathrm{X}$ receptor and retinoid $\mathrm{X}$ receptor heterodimer mediates apolipoprotein A1 expression. Matrix Biol. 2005, 24, 35-44.

79. Woods, A.; James, C.G.; Wang, G.; Dupuis, H.; Beier, F. Control of chondrocyte gene expression by actin dynamics: A novel role of cholesterol/Ror- $\alpha$ signalling in endochondral bone growth. J. Cell. Mol. Med. 2009, 13, 3497-3516.

80. Kakinuma, T.; Yasuda, T.; Nakagawa, T.; Hiramitsu, T.; Akiyoshi, M.; Akagi, M.; Sawamura, T.; Nakamura, T. Lectin-like oxidized low-density lipoprotein receptor 1 mediates matrix metalloproteinase 3 synthesis enhanced by oxidized low-density lipoprotein in rheumatoid arthritis cartilage. Arthritis Rheum. 2004, 50, 3495-3503.

81. Akagi, M.; Kanata, S.; Mori, S.; Itabe, H.; Sawamura, T.; Hamanishi, C. Possible involvement of the oxidized low-density lipoprotein/lectin-like oxidized low-density lipoprotein receptor-1 system in pathogenesis and progression of human osteoarthritis. Osteoarthr. Cartil. 2007, 15, 281-290.

82. Zushi, S.; Akagi, M.; Kishimoto, H.; Teramura, T.; Sawamura, T.; Hamanishi, C. Induction of bovine articular chondrocyte senescence with oxidized low-density lipoprotein through lectin-like oxidized low-density lipoprotein receptor 1. Arthritis Rheum. 2009, 60, 3007-3016.

83. Kishimoto, H.; Akagi, M.; Zushi, S.; Teramura, T.; Onodera, Y.; Sawamura, T.; Hamanishi, C. Induction of hypertrophic chondrocyte-like phenotypes by oxidized LDL in cultured bovine articular chondrocytes through increase in oxidative stress. Osteoarthr. Cartil. 2010, 18, 1284-1290.

84. Nishimura, S.; Akagi, M.; Yoshida, K.; Hayakawa, S.; Sawamura, T.; Munakata, H.; Hamanishi, C. Oxidized low-density lipoprotein (ox-LDL) binding to lectin-like ox-LDL receptor-1 (LOX-1) in cultured bovine articular chondrocytes increases production of intracellular reactive oxygen species (ROS) resulting in the activation of NF-кB. Osteoarthr. Cartil. 2004, 12, 568-576. 
85. Akagi, M.; Ueda, A.; Teramura, T.; Kanata, S.; Sawamura, T.; Hamanishi, C. Oxidized LDL binding to LOX-1 enhances MCP-1 expression in cultured human articular chondrocytes. Osteoarthr. Cartil. 2009, 17, 271-275.

86. Lippiello, L. Lipid and cell metabolic changes associated with essential fatty acid enrichment of articular chondrocytes. Proc. Soc. Exp. Biol. Med. 1990, 195, 282-287.

87. Curtis, C.L.; Hughes, C.E.; Flannery, C.R.; Little, C.B.; Harwood, J.L.; Caterson, B. N-3 fatty acids specifically modulate catabolic factors involved in articular cartilage degradation. J. Biol. Chem. 2000, 275, 721-724.

88. Zainal, Z.; Longman, A.J.; Hurst, S.; Duggan, K.; Caterson, B.; Hughes, C.E.; Harwood, J.L. Relative efficacies of $\omega-3$ polyunsaturated fatty acids in reducing expression of key proteins in a model system for studying osteoarthritis. Osteoarthr. Cartil. 2009, 17, 896-905.

89. Sabatini, M.; Rolland, G.; Léonce, S.; Thomas, M.; Lesur, C.; Pérez, V.; de Nanteuil, G.; Bonnet, J. Effects of ceramide on apoptosis, proteoglycan degradation, and matrix metalloproteinase expression in rabbit articular cartilage. Biochem. Biophys. Res. Commun. 2000, 267, 438-444.

90. Gilbert, S.J.; Blain, E.J.; Duance, V.C.; Mason, D.J. Sphingomyelinase decreases type II collagen expression in bovine articular cartilage chondrocytes via the ERK signaling pathway. Arthritis Rheum. 2008, 58, 209-220.

91. Gilbert, S.J.; Blain, E.J.; Jones, P.; Duance, V.C.; Mason, D.J. Exogenous sphingomyelinase increases collagen and sulphated glycosaminoglycan production by primary articular chondrocytes: An in vitro study. Arthritis Res. Ther. 2006, doi:10.1186/ar1961.

92. Simonaro, C.M.; Sachot, S.; Ge, Y.; He, X.; Deangelis, V.A.; Eliyahu, E.; Leong, D.J.; Sun, H.B.; Mason, J.B.; Haskins, M.E.; et al. Acid ceramidase maintains the chondrogenic phenotype of expanded primary chondrocytes and improves the chondrogenic differentiation of bone marrow-derived mesenchymal stem cells. PLoS One 2013, 8, e62715.

93. Laplante, M.; Sabatini, D.M. mTOR signaling at a glance. J. Cell Sci. 2009, 122, 3589-3594.

94. André, C.; Cota, D. Coupling nutrient sensing to metabolic homoeostasis: The role of the mammalian target of rapamycin complex 1 pathway. Proc. Nutr. Soc. 2012, 71, 502-510.

95. Mordier, S.; Iynedjian, P.B. Activation of mammalian target of rapamycin complex 1 and insulin resistance induced by palmitate in hepatocytes. Biochem. Biophys. Res. Commun. 2007, 362, 206-211.

96. Rivas, D.A.; Yaspelkis, B.B.; Hawley, J.A.; Lessard, S.J. Lipid-induced mTOR activation in rat skeletal muscle reversed by exercise and 5 '-aminoimidazole-4-carboxamide-1- $\beta$-D-ribofuranoside. J. Endocrinol. 2009, 202, 441-451.

97. Wen, Z.-H.; Su, Y.-C.; Lai, P.-L.; Zhang, Y.; Xu, Y.-F.; Zhao, A.; Yao, G.-Y.; Jia, C.-H.; Lin, J.; $\mathrm{Xu}, \mathrm{S}$; et al. Critical role of arachidonic acid-activated mTOR signaling in breast carcinogenesis and angiogenesis. Oncogene 2013, 32, 160-170.

98. Pattingre, S.; Bauvy, C.; Levade, T.; Levine, B.; Codogno, P. Ceramide-induced autophagy: To junk or to protect cells? Autophagy 2009, 5, 558-560.

99. Mathis, A.S.; Jin, S.; Friedman, G.S.; Peng, F.; Carl, S.M.; Knipp, G.T. The pharmacodynamic effects of sirolimus and sirolimus-calcineurin inhibitor combinations on macrophage scavenger and nuclear hormone receptors. J. Pharm. Sci. 2007, 96, 209-222. 
100. Xu, J.; Dang, Y.; Ren, Y.R.; Liu, J.O. Cholesterol trafficking is required for mTOR activation in endothelial cells. Proc. Natl. Acad. Sci. USA 2010, 107, 4764-4769.

101. Srinivas, V.; Bohensky, J.; Shapiro, I.M. Autophagy: A new phase in the maturation of growth plate chondrocytes is regulated by HIF, mTOR and AMP kinase. Cells Tissues Organs 2009, 189, 88-92.

102. Phornphutkul, C.; Wu, K.-Y.; Auyeung, V.; Chen, Q.; Gruppuso, P.A. mTOR signaling contributes to chondrocyte differentiation. Dev. Dyn. 2008, 237, 702-712.

103. Caramés, B.; Taniguchi, N.; Otsuki, S.; Blanco, F.J.; Lotz, M. Autophagy is a protective mechanism in normal cartilage, and its aging-related loss is linked with cell death and osteoarthritis. Arthritis Rheum. 2010, 62, 791-801.

104. Caramés, B.; Hasegawa, A.; Taniguchi, N.; Miyaki, S.; Blanco, F.J.; Lotz, M. Autophagy activation by rapamycin reduces severity of experimental osteoarthritis. Ann. Rheum. Dis. 2012, 71, 575-581.

105. Sasaki, H.; Takayama, K.; Matsushita, T.; Ishida, K.; Kubo, S.; Matsumoto, T.; Fujita, N.; Oka, S.; Kurosaka, M.; Kuroda, R. Autophagy modulates osteoarthritis-related gene expression in human chondrocytes. Arthritis Rheum. 2012, 64, 1920-1928.

(C) 2013 by the authors; licensee MDPI, Basel, Switzerland. This article is an open access article distributed under the terms and conditions of the Creative Commons Attribution license (http://creativecommons.org/licenses/by/3.0/). 